\title{
El conocimiento del profesorado para enseñar probabilidad: Un análisis global desde el modelo del Conocimiento Didáctico-Matemático
}

Claudia Vásquez Ortiz, Pontificia Universidad Católica de Chile, sede Villarrica (Chile).

Ángel Alsina Pastells, Universidad de Girona (España).

Recibido el 19 de Diciembre de 2014; aceptado el 9 de Marzo de 2015

El conocimiento del profesorado para enseñar probabilidad: Un análisis global desde el modelo del Conocimiento Didáctico - Matemático

\section{Resumen}

En este artículo se presenta un análisis global de los resultados obtenidos en un estudio exploratorio sobre el conocimiento didáctico-matemático para enseñar probabilidad de los profesores de Educación Primaria, fundamentado en el modelo del Conocimiento Didáctico-Matemático (CDM). Para evaluar las distintas facetas que involucra este tipo de conocimiento se analizan las prácticas matemáticas de 93 profesores de Educación Primaria en activo, a partir de las respuestas al cuestionario de respuesta abierta CDM-Probabilidad. Los resultados evidencian un conocimiento didáctico-matemático muy insuficiente para las distintas categorías que lo componen. Se concluye que es urgente elaborar programas de intervención que permitan desarrollar el conocimiento didácticomatemático sobre probabilidad de los profesores de Educación Primaria.

Palabras clave: probabilidad; profesorado; educación primaria; conocimiento didácticomatemático.

O conhecimento dos professores para ensinar probabilidade: Uma análise global a partir do modelo de Conhecimento Didático-Matemático

\section{Resumo}

Este artigo apresenta uma análise global dos resultados de um estudo exploratório sobre o conhecimento didático-matemático dos professores da Educação Primária para ensinar probabilidade, baseado no modelo do Conhecimento Didático-Matemático. Para avaliar as diferentes facetas que envolvem este tipo de conhecimento analisaram-se as práticas matemáticas de 93 professores da Educação Primária através das respostas ao questionário de resposta aberta CDMProbabilidade. Os resultados mostram um conhecimento didático-matemático insuficiente para as diferentes categorias que o compõem. Conclui-se que é urgente realizar programas de intervenção que permitam aos professores da Educação Primária adquirir conhecimento matemático sobre probabilidade.

Palavras-chave: profesores; educação primária; conhecimento didático-matemático; probabilidade.

Para citar: Vásquez, C. \& Alsina, A. (2015). El conocimiento del profesorado para enseñar probabilidad: Un análisis global desde el modelo del Conocimiento Didáctico-Matemático. Avances de Investigación en Educación Matemática, 7, 27 - 48.

(C) Sociedad Española de Investigación en Educción Matemática (SEIEM). www.seiem.es 


\section{Teachers' knowledge for teaching probability: A global analysis based on Didactic- Mathematical Knowledge model}

\section{Abstract}

This article presents a global analysis of the results obtained in an exploratory assessment of Primary School teachers' mathematical knowledge for teaching probability, based on the DidacticMathematical Knowledge model. In order to evaluate the different facets which comprise this kind of knowledge, the mathematical practices of 93 in-service Primary Education teachers were analized on the basis of their answers to the CDM-Probability open-ended questionnaire. The results show an inadequate mathematical knowledge for teaching for different categories that compose it. We conclude that there is an urgent need to elaborate intervention programmes for teachers to develop their mathematical knowledge for teaching probability.

Key words: probability; teachers; primary education; mathematical and didactic knowledge of teacher.

La connaissance des enseignants pour enseigner probabilité: Une analyse globale à partir du modèle des connaissances pédagogiques-mathématicien

\section{Résumé}

Cet article présente une analyse globale des résultats d'une étude exploratoire sur les connaissances pédagogiques-mathématicien des enseignants de l'école primaire pour enseigner probabilité, sur la base du modèle des Connaissances Pédagogiques-Mathématicien. Pour évaluer les différents aspects concernant cette connaissance, nous avons analysé les pratiques mathématiques de 93 enseignants du primaire avec le questionnaire CDM-Probabilité. Les résultats obtenus avec ce questionnaire ouvert indiquent une connaissance pédagogique-mathématicien très insuffisante pour les différentes catégories qui le composent. Nous concluons qu'il est urgent d'élaborer des programmes d'intervention a fin de développer les connaissances pédagogiques-mathématicien des enseignants de l'école primaire pour enseigner probabilité.

Mots-clés: probabilité; enseignants; enseignement primaire; connaissances pédagogiquemathématicien.

\section{Introducción}

Durante las últimas décadas la probabilidad se ha incorporado con fuerza en el currículo de Educación Primaria con el objeto de promover un enfoque experimental que proporcione a los estudiantes una experiencia estocástica desde las primeras edades (NCTM, 1989; NCTM, 2003; MEC, 2007; CCSSI, 2010; MINEDUC, 2012).

Esta transformación curricular representa un verdadero desafío, ya que muchos profesores no han recibido una formación sobre la probabilidad que les permita llevar a cabo una enseñanza idónea en las aulas, es decir, una formación en la que "el profesor sea capaz de comprender lo que los estudiantes conocen y necesitan aprender y, en consecuencia, les desafía y apoya para aprender bien los nuevos conocimientos" (NCTM, 2003, p. 17).

En el caso de Chile, las cifras internacionales revelan carencias en la educación en general y de manera particular en matemáticas. El informe 2010 de la OCDE, por ejemplo, manifiesta que Chile debe encauzar sus esfuerzos hacia la mejora de la formación de profesores en todos los niveles educativos, sobre todo en profesores de Educación Primaria puesto que "reciben una formación general, y los conocimientos que adquieren sobre las materias no resultan suficientes ni siquiera para los cursos iniciales" (OCDE, 2010, p.10). Asimismo, en el Reporte de Competitividad Global 2011-2012 del World Economic Forum, en un ranking de 142 países, Chile se 
encuentra en el número 87 en calidad general de la educación, y en el número 124 en calidad de la educación de matemáticas y ciencias.

A partir de estos datos genéricos, en este estudio se analizan los conocimientos del profesorado de Educación Primaria sobre probabilidad, para poder valorar si estos conocimientos son adecuados y suficientes para la aplicación de los nuevos currículos, puesto que no hay que olvidar que finalmente es el profesorado quien lo pone en funcionamiento. Para realizar dicho análisis, en este trabajo asumimos el modelo del Conocimiento Didáctico-Matemático CDM (Godino, 2009; Godino \& Pino-Fan, 2013; Pino-Fan, Godino \& Font, 2013), fundamentado en el Enfoque Ontosemiótico del Conocimiento y la Instrucción Matemática (EOS) (Godino, 2002; Godino, Batanero \& Font, 2007). A partir de las herramientas teóricas que proporciona este modelo, hemos construido un instrumento para evaluar el conocimiento didáctico-matemático para enseñar probabilidad que se ha administrado a un grupo de 93 profesores chilenos de Educación Primaria en activo.

\section{Fundamentos}

Los campos de estudio en educación matemática que fundamentan teóricamente la investigación que pretendemos realizar son de dos tipos: el conocimiento didácticomatemático del profesorado de matemáticas y las investigaciones previas sobre el conocimiento del profesorado de primaria para enseñar probabilidad.

\subsection{El conocimiento didáctico-matemático del profesorado de matemáticas}

El dominio del profesor en relación a los contenidos que debe enseñar es un elemento clave con efectos directos en el aprendizaje de sus estudiantes, pues un profesor no debe enseñar lo que no sabe bien. En este sentido, Godino (2009) realiza una reestructuración de los componentes del modelo del Conocimiento Matemático para la Enseñanza, conocido como MKT (Hill, Ball \& Schilling, 2008), y explicita el vínculo e interacción con las seis facetas implicadas en los procesos de enseñanza y aprendizaje de las matemáticas (epistémica, cognitiva, afectiva, interaccional, mediacional y ecológica). Dicha reestructuración propone tres categorías globales del conocimiento sobre el contenido matemático (descritas posteriormente en Pino-Fan, Godino \& Font, 2013):

1. Conocimiento común del contenido: se refiere a los conocimientos matemáticos, no necesariamente orientados a la enseñanza, que el profesor debe poner en juego para resolver situaciones problemáticas en relación a un tema de matemáticas de un nivel educativo concreto en el que se enmarca la situación problema. Se analiza a través de la faceta epistémica.

2. Conocimiento avanzado del contenido: es también un conocimiento de tipo matemático que se refiere a que el profesor, además de saber resolver situaciones problemáticas sobre un determinado tema y nivel, debe poseer conocimientos más avanzados del currículo. Se analiza a través de la faceta epistémica.

3. Conocimiento especializado: se refiere al conocimiento que diferencia al profesor de otras personas que saben matemáticas. Además de implicar conocimiento común y parte del conocimiento avanzado, "debe incluir la pluralidad de significados del objeto, la diversidad de configuraciones de objetos y procesos inherentes a tales significados y las necesarias articulaciones inherentes entre los mismos" (Pino-Fan et 
al., 2013, p. 6). Este conocimiento es interpretado desde la faceta epistémica, e incluye cuatro subcategorías:

- Conocimiento del contenido especializado: se fundamenta en las facetas implicadas en los procesos de instrucción matemática, y se refiere a que un profesor no solo debe ser capaz de resolver situaciones problemáticas en relación a un determinado contenido aplicando diversos significados parciales vinculados al objeto matemático en cuestión, diferentes tipos de representaciones, conceptos, proposiciones, procedimientos y argumentos, sino que además debe identificar los conocimientos puestos en juego (elementos lingüísticos, conceptos, propiedades, procedimientos y argumentos) en la resolución de un problema.

- Conocimiento del contenido en relación con los estudiantes: se fundamenta en la faceta cognitiva y afectiva, y se refiere a la reflexión sistemática, por parte del profesor, sobre el aprendizaje de los estudiantes, lo que implica la capacidad para: describir los tipos de configuraciones cognitivas que los estudiantes han desarrollado al resolver la situación problemática propuesta, describir los principales tipos de conflictos de aprendizaje en la resolución de un cierto tipo de situaciones problemáticas, formular cuestiones que permitan explicitar los significados personales al resolver cierto tipo de situaciones problemáticas, así como describir estrategias que se pueden implementar para promover que los estudiantes se involucren en la solución de situaciones problemáticas o en el estudio de un determinado tema (Godino, 2009).

- Conocimiento del contenido en relación con la enseñanza: se fundamenta en la faceta interaccional y mediacional, y se refiere a la reflexión sistemática, por parte del profesor, sobre las relaciones entre la enseñanza y el aprendizaje, y la identificación de las consecuencias que pueden tener sobre el aprendizaje los modelos de gestión de la clase (Godino, 2009).

- Conocimiento del contenido en relación con el currículo: se fundamenta en la faceta ecológica, y se refiere al contexto en el que se desarrolla la práctica de enseñanza-aprendizaje.

Para el análisis de estas categorías se emplean herramientas teóricas del EOS: por ejemplo, para el conocimiento común del contenido, el conocimiento avanzado del contenido y el conocimiento del contenido especializado se puede usar la noción de configuración de objetos y procesos, por medio de la "Guía para el reconocimiento de objetos y procesos" (Godino, Gonzato \& Fernández, 2010); mientras que las categorías restantes pueden ser analizadas mediante las herramientas teóricas y metodológicas que el EOS entrega para las distintas facetas: cognitiva y afectiva (conocimiento del contenido en relación a los estudiantes), interaccional y mediacional (conocimiento del contenido en relación a la enseñanza), y ecológica y epistémica (conocimiento del contenido en relación con el currículo), sin olvidar que por medio de la "Guía para el enunciado de consignas" (Godino, 2009) es posible orientar la formulación de ítems de evaluación que permiten obtener información sobre el conocimiento didáctico-matemático del profesor.

\subsection{Investigaciones previas sobre el conocimiento del profesorado de primaria para enseñar probabilidad}

Las investigaciones al respecto son muy escasas, se focalizan sobre todo en profesores en formación y se centran principalmente en determinar si los futuros 
profesores cuentan o no con los conocimientos suficientes para llevar a cabo una enseñanza idónea de la probabilidad.

Una de las primeras investigaciones sobre el conocimiento del profesorado para enseñar probabilidad fue realizada por Begg y Edward (1999), quienes al solicitar a 22 profesores responder a tres situaciones relacionadas con ideas básicas de aleatoriedad, sucesos equiprobables e independencia, detectan una escasa comprensión. Watson (2001), aplica una encuesta sobre probabilidad y su enseñanza a 15 profesores de primaria y 28 de secundaria, y concluye que el profesorado de secundaria presenta mayor confianza, mientras que el profesorado de primaria tiende a emplear una mentalidad determinista centrada en un enfoque clásico orientado al cálculo de probabilidades a priori, aspectos ratificados por Pereira-Mendoza (2002).

Estudios con futuros profesores de primaria indican resultados similares, como el realizado por Azcárate, Cardeñoso y Porlán (1998), quienes estudian la noción de suceso aleatorio en 57 profesores en formación, encontrando que en su mayoría no reconocen la aleatoriedad. No obstante, perciben correctamente la multiplicidad de posibilidades y el carácter impredecible de los posibles resultados. Batanero, Godino y Cañizares (2005) evalúan la presencia de sesgos en el razonamiento probabilístico en 132 estudiantes para maestro, y los resultados obtenidos revelan que un porcentaje importante razona según la heurística de la representatividad (Tversky \& Kahneman, 1982). Además, la gran mayoría presenta el sesgo de equiprobabilidad descrito por Lecoutre (1992) y Lecoutre y Durand (1988), y un pequeño porcentaje muestra también el sesgo “outcome approach” (Konold, 1991).

El estudio de Ortiz, Mohamed, Batanero, Serrano y Rodríguez (2006), con 102 futuros profesores, evidencia cierta mejora con respecto a investigaciones anteriores, aun cuando se observa una falta de razonamiento proporcional para la resolución de algunos problemas, así como la influencia de factores del problema que inducen a la asignación de probabilidades subjetivas. Mohamed (2012) evalúa el conocimiento matemático para la enseñanza, según el modelo de Ball y colaboradores, de 283 futuros profesores en relación a la idea de juego equitativo, a través de sus respuestas a un problema extraído de un libro de texto de Educación Primaria. Los resultados evidencian un escaso conocimiento, puesto que la mayoría no identifica ni aplica correctamente este conocimiento.

Gómez (2014) evalúa el conocimiento matemático para la enseñanza de 157 futuros profesores y la evolución de su conocimiento del contenido como consecuencia de una acción formativa. Parte de este conocimiento es analizado previamente en Batanero, Gómez, Serrano y Contreras (2012) a partir de un problema sobre percepción subjetiva de la aleatoriedad tomado de Green (1983). Los resultados muestran una mezcla de intuiciones y creencias correctas e incorrectas sobre la forma de percibir la aleatoriedad. Asimismo, se observan sesgos como la falacia del jugador o el enfoque en los resultados, además de concepciones erróneas sobre equiprobabilidad o falta de comprensión de independencia de sucesos. Posteriormente, Gómez, Batanero y Contreras (2013) analizan las respuestas vinculadas al conocimiento común del contenido, conocimiento especializado del contenido y conocimiento del contenido y los estudiantes sobre probabilidad desde su significado frecuencial. Las respuestas de los 157 futuros profesores vinculadas al conocimiento común del contenido evidencian un pobre razonamiento probabilístico con predominio de diversos sesgos probabilísticos y estrategias aritméticas. Sin embargo, en lo que respecta al conocimiento especializado del contenido y el 
conocimiento del contenido en relación a los estudiantes, los resultados son un poco mejores, producto de una metodología de análisis colectivo de las propias respuestas y actividades de experimentación. Los datos señalan la utilidad de dicha metodología para el desarrollo del conocimiento matemático para la enseñanza de la probabilidad en futuros profesores de primaria. Finalmente, Batanero, Gómez, Contreras y Díaz (2015) analizan las tareas propuestas en el estudio anterior y evalúan algunos componentes del conocimiento matemático para la enseñanza. Los resultados evidencian un conocimiento común insuficiente con una fuerte presencia del sesgo de la equiprobabilidad, y al igual que en los estudios anteriores, un pobre conocimiento especializado del contenido. No obstante, los resultados sobre el conocimiento del contenido y de los estudiantes son bastante alentadores, presentando una mejora sustancial en relación a la capacidad para justificar los errores presentes en respuestas hipotéticas o ficticias.

Tomando en consideración los estudios anteriores, nuestro estudio se orienta a diagnosticar las principales dificultades que presenta el profesorado de primaria en activo en las distintas categorías que componen el conocimiento didáctico-matemático sobre probabilidad, para poder diseñar estrategias de intervención apropiadas que den lugar a un conocimiento profundo y acabado del contenido a enseñar y cómo enseñarlo. Con este propósito, se diseña el Cuestionario CDM-Probabilidad (Anexo 1) descrito en Vásquez y Alsina (en prensa). El cuestionario está formado por 7 ítems, algunos de los cuales son de elaboración propia y otros se han reformulado a partir de las investigaciones de Cañizares (1997), Green (1983) y Fischbein y Gazit (1984). Por medio de estos ítems se abordan los contenidos que conforman nuestro significado de referencia sobre la probabilidad (Tabla 1) adoptado a partir del análisis de las orientaciones curriculares tanto internacionales como chilenas (Vásquez y Alsina, 2014).

Tabla 1. Contenidos que se espera movilizar en el conjunto de items que conforman el cuestionario del conocimiento didáctico-matemático sobre probabilidad.

\begin{tabular}{|c|c|c|c|c|c|c|c|}
\hline \multirow{2}{*}{ Contenidos } & \multicolumn{7}{|c|}{ Ítems } \\
\hline & 1 & 2 & 3 & 4 & 5 & 6 & 7 \\
\hline Experimento y suceso aleatorio & $\mathrm{x}$ & $\mathrm{x}$ & $\mathrm{x}$ & $\mathrm{x}$ & & $\mathrm{x}$ & $\mathrm{x}$ \\
\hline Espacio muestral & $\mathrm{x}$ & $\mathrm{x}$ & $\mathrm{x}$ & $\mathrm{x}$ & $\mathrm{x}$ & $\mathrm{x}$ & $\mathrm{x}$ \\
\hline Posibilidad de ocurrencia (seguro, posible, imposible) & & $\mathrm{x}$ & $\mathrm{x}$ & & $\mathrm{x}$ & & \\
\hline Significados de la probabilidad & $\mathrm{x}$ & $\mathrm{x}$ & $\mathrm{x}$ & $\mathrm{x}$ & $\mathrm{x}$ & $\mathrm{x}$ & $\mathrm{x}$ \\
\hline Cálculo de probabilidad & $\mathrm{x}$ & $\mathrm{x}$ & & $\mathrm{x}$ & & $\mathrm{x}$ & $\mathrm{x}$ \\
\hline Comparación de probabilidades & & $\mathrm{x}$ & & $\mathrm{x}$ & & $\mathrm{x}$ & \\
\hline Independencia de sucesos & $\mathrm{x}$ & & & & $\mathrm{x}$ & & $\mathrm{x}$ \\
\hline Equiprobabilidad & $\mathrm{x}$ & & & $\mathrm{x}$ & & $\mathrm{x}$ & $\mathrm{x}$ \\
\hline
\end{tabular}

En este trabajo, pues, presentamos un análisis global de los resultados obtenidos en un estudio exploratorio sobre el conocimiento didáctico-matemático para enseñar probabilidad de los profesores de Educación Primaria en activo.

\section{Método}

En el estudio participaron 93 profesores de Educación Primaria de 22 centros educativos que imparten clases de matemáticas de $1^{\circ}$ a $6^{\circ}$ en la región de La 
Araucanía (Chile). Estos profesores asistieron a un Seminario-Taller gratuito sobre "Enseñanza de la probabilidad en la Educación Básica" realizado por una universidad del sur de Chile. Los profesores accedieron a responder voluntariamente el cuestionario CDM-Probabilidad después de la firma del consentimiento informado y de la revisión de las instrucciones.

Los profesores participantes imparten docencia en distintos tipos de establecimientos educacionales: municipales $(33,3 \%)$, particulares subvencionados $(59,1 \%)$ y particulares privados $(7,5 \%)$. En cuanto a la distribución según el género, hay 68 mujeres $(73,1 \%)$ y 25 hombres $(26,9 \%)$. Respecto a la especialización, un $76,3 \%$ no tiene especialidad, un $15,1 \%$ tiene especialidad en matemáticas y un $8,6 \%$ tiene otra especialidad. En relación a los años de experiencia, la gran mayoría de los participantes $(46,2 \%)$ tiene menos de 3 años de experiencia enseñando matemáticas en Educación Primaria, el 21,5\% tiene entre 3 y 5 años, un 17,2\% tiene entre 5 y 10 años y tan solo un $15,1 \%$ tiene más de 10 años de experiencia.

Otro dato de interés es que al preguntarles si se sienten preparados para enseñar probabilidad, un $5,4 \%$ declara sentirse muy preparado, un $60,2 \%$ medianamente preparado y un $34,4 \%$ no se siente preparado. Al preguntarles si enseñan probabilidad en sus cursos, un $68,8 \%$ responde afirmativamente, un $28 \%$ no lo enseña y un $3,2 \%$ no responde.

Finalmente se les pregunta si durante su formación inicial como profesores de Educación Primara han recibido formación sobre probabilidad: un 31,2\% afirma que sí y un $68,8 \%$ declara no haber recibido ninguna formación. Asimismo, se les pregunta si han recibido formación en didáctica de la probabilidad, y el 100\% declara que no.

Una vez recogidos los datos, se codifican según el grado de corrección de las respuestas, asignando las siguientes puntuaciones: " 2 " si la respuesta es correcta, " 1 " si es parcialmente correcta y " 0 " si es incorrecta. Los criterios para definir a cuál de las tres categorías pertenece la respuesta se explicitaron por medio de un rubrica. A partir de esta codificación se analizaron aspectos psicométricos del instrumento, de los que a continuación se describen la fiabilidad de la consistencia interna del cuestionario y los índices de dificultad de los ítems que lo componen.

\subsection{Fiabilidad de la consistencia interna del cuestionario}

Las respuestas al cuestionario permiten inferir aspectos del conocimiento didáctico-matemático sobre probabilidad, al tratarse de un concepto que no se puede observar directamente. Por esta razón, se analiza la fiabilidad de la consistencia interna del instrumento, es decir, la estabilidad en las puntuaciones que proporciona si se administrara en repetidas ocasiones al mismo grupo de profesores. Con este propósito aplicamos el coeficiente alfa de Cronbach, obteniendo un valor 0,760 , que es aceptable y sugiere que el instrumento permite realizar mediciones estables y consistentes. Aunque el valor no es excesivamente elevado, es suficiente para nuestro propósito, puesto que el cuestionario no es homogéneo en cuanto a la variedad de aspectos y contenidos que evalúa.

\section{2.Índice de dificultad de los ítems}

Este índice se entiende como la proporción entre "número de sujetos que aciertan el ítem/número de sujetos que han intentado resolver el ítem” (Muñiz, 1994), variando 
sus valores entre 0 y 1 , donde " 0 " indica que el ítem tiene un alto grado de dificultad y "1" un grado de máxima facilidad. Para el cálculo del índice de dificultad (I.D.) clasificamos las respuestas en correctas e incorrectas, las respuestas en blanco no se consideraron.

La Tabla 2 presenta los I.D. para cada uno de los ítems que componen el cuestionario, que clasificamos como alto: entre $0,0-0,167$; medio: entre $0,301-0,402$; y bajo: 0,622 . El promedio fue 0,127 , que indica muy pocos conocimientos de los participantes.

Tabla 2. Índice de dificultad de los items de acuerdo con el tipo de conocimiento evaluado.

\begin{tabular}{|c|c|c|c|c|c|c|c|c|}
\hline \multirow{2}{*}{ Ítem } & & \multicolumn{7}{|c|}{ Tipo de conocimiento evaluado } \\
\hline & & Í.D. & $\begin{array}{c}\text { Común } \\
\text { del } \\
\text { contenido }\end{array}$ & $\begin{array}{c}\text { Especializado } \\
\text { del } \\
\text { contenido } \\
\end{array}$ & $\begin{array}{c}\text { Avanzado } \\
\text { del } \\
\text { contenido }\end{array}$ & $\begin{array}{c}\text { Contenido } \\
\text { y } \\
\text { estudiante } \\
\end{array}$ & $\begin{array}{l}\text { Contenido y } \\
\text { enseñanza }\end{array}$ & Currículo \\
\hline \multirow{5}{*}{1} & & 0,069 & $\mathrm{x}$ & & & & & \\
\hline & & 0,301 & & & & $\mathrm{x}$ & & \\
\hline & & 0,000 & & $\mathrm{x}$ & & & & \\
\hline & & 0,300 & & & & $\mathrm{x}$ & & \\
\hline & & 0,079 & & & & & $\mathrm{x}$ & \\
\hline \multirow{4}{*}{2} & & 0,402 & $\mathrm{x}$ & & & & & \\
\hline & & 0,013 & & $\mathrm{x}$ & & $\mathrm{x}$ & & \\
\hline & & 0,014 & & & & & & \\
\hline & & 0,167 & & & & & $\mathrm{x}$ & \\
\hline \multirow{3}{*}{3} & & 0,061 & $\mathrm{x}$ & & & & & \\
\hline & & 0,031 & & $\mathrm{x}$ & & & & \\
\hline & & 0,043 & & & & & $\mathrm{x}$ & \\
\hline \multirow{4}{*}{4} & & 0,330 & $\mathrm{x}$ & & & $\mathrm{x}$ & & \\
\hline & & 0,027 & & $\mathrm{x}$ & & & & \\
\hline & & 0,044 & & & & $\mathrm{x}$ & & \\
\hline & 1 & 0,048 & & & & & $\mathrm{x}$ & \\
\hline 5 & & 0,141 & $\mathrm{x}$ & & & $\mathrm{x}$ & & \\
\hline 6 & & 0,622 & $\mathrm{x}$ & & & $\mathrm{x}$ & & \\
\hline \multirow{4}{*}{7} & & 0,040 & $\mathrm{x}$ & & & & & \\
\hline & 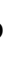 & 0,040 & & & & & & $\mathrm{x}$ \\
\hline & & 0,030 & & & & & $\mathrm{x}$ & \\
\hline & 1 & 0,000 & & & $x$ & & & \\
\hline
\end{tabular}

En la Figura 1 se observa que el cuestionario presentó un nivel de dificultad bastante alto que osciló entre un $0 \%$ y un $62 \%$. La mediana tiene un valor muy bajo $(0,048)$, la distribución de los índices es bastante asimétrica y sus valores se encuentran concentrados en los valores más bajos (entre 0 y 0,0305 ), lo que muestra que el cuestionario resultó bastante difícil para los profesores. Por último, se observa que existen dos outliers que denotan los índices de menor dificultad $(0,402$ y 0,622$)$. Estos valores se vinculan al conocimiento común del contenido sobre el cálculo y la comparación de probabilidades de sucesos elementales no equiprobables en un experimento simple, y al conocimiento común del contenido y el conocimiento del contenido en relación con los estudiantes sobre la comparación de probabilidades y la noción de juego equitativo respectivamente. 


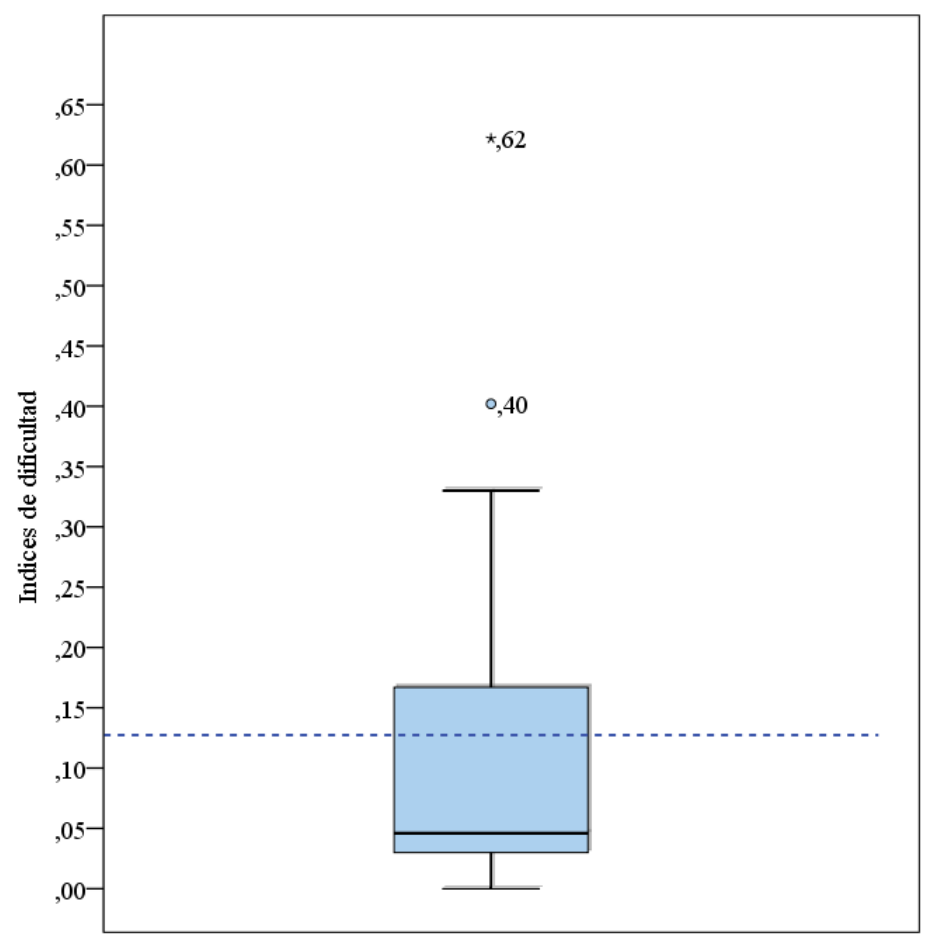

Figura 1. Distribución y media de los índices de dificultad.

\section{Resultados}

Una vez analizadas las características psicométricas del instrumento se realizó, en primer lugar, un análisis de las puntuaciones totales del cuestionario; y en segundo lugar, una primera aproximación a nivel global de los resultados obtenidos en relación a las tres categorías del conocimiento didáctico-matemático.

\subsection{Puntuación total del cuestionario}

Para el análisis de la puntuación total del cuestionario se categorizaron las respuestas de los 93 profesores según el grado de corrección indicado. De acuerdo con estas puntuaciones, en el cuestionario CDM-Probabilidad es posible obtener una puntuación máxima de 44 puntos, puesto que se compone de 7 ítems que abordan 22 preguntas en total. Los puntajes totales obtenidos fluctúan entre 1 y 28 puntos, de lo que se desprende que ningún profesor resolvió correctamente el cuestionario en su totalidad. Además, la puntuación media fue 10,92 puntos (siendo la media teórica 22 puntos) con una desviación típica de 5,398 puntos, lo cual es muy bajo dado que dicha puntuación considera tanto las respuestas correctas como las parcialmente correctas.

En la Figura 2 se observa un valor bajo de la mediana (11 puntos) y los valores de las puntuaciones totales que se encuentran más concentradas en la zona superior de la caja. Por otro lado, las amplitudes de los bigotes superior e inferior son similares, lo que indica cierta similitud entre los extremos de la distribución de los puntajes totales. Además se observa la presencia de dos outliers que corresponden a observaciones extremas que se alejan del grueso de los datos, en este caso dos profesores que obtuvieron una puntuación total de 27 y 28 puntos, de un total de 44 puntos posibles. En resumen, estos resultados indican que estos profesores de primaria presentan 
grandes dificultades en relación al conocimiento didáctico-matemático para enseñar probabilidad.

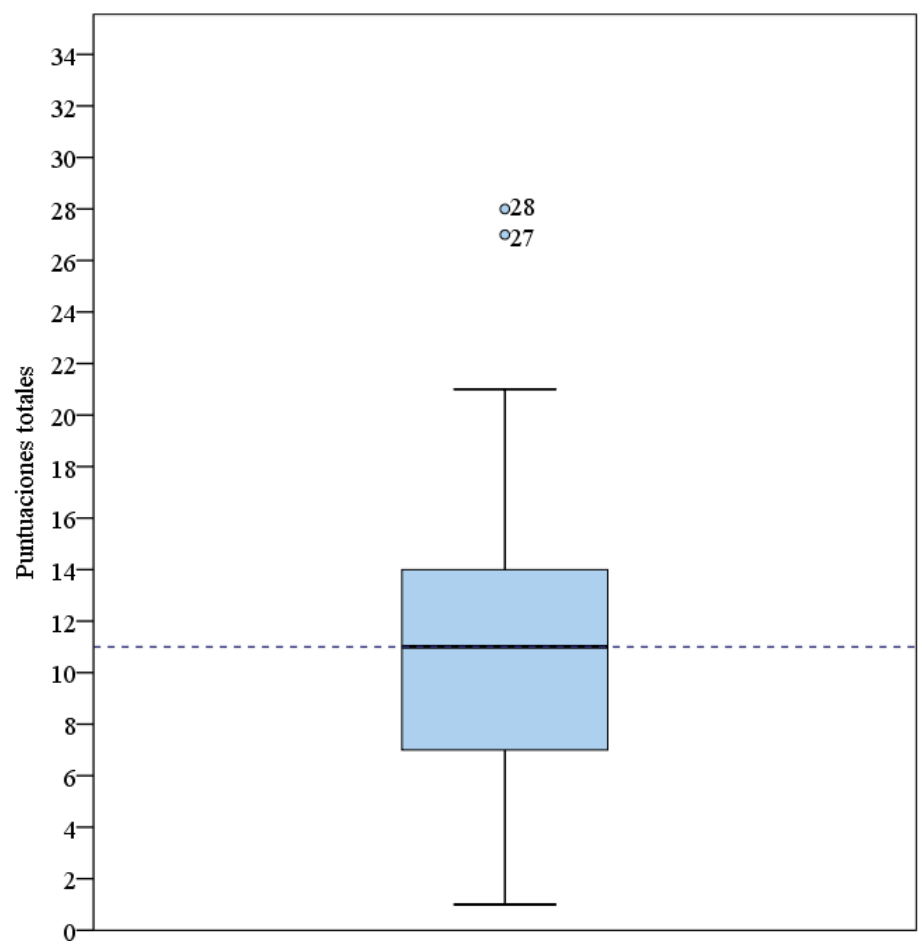

Figura 2. Distribución de las puntuaciones totales y puntuación media del cuestionario.

\subsection{Comparación de resultados en componentes del conocimiento didáctico- matemático para enseñar probabilidad}

Para establecer una comparación entre las distintas categorías de conocimiento didáctico-matemático para enseñar probabilidad, recodificamos las puntuaciones totales para cada tipo de conocimiento de acuerdo a una escala normada de 0 a 100 , pues en cada una de las categorías el número de ítems difiere. En la Figura 4 se comparan estas puntuaciones, que evidencian que estos profesores poseen un conocimiento didáctico-matemático sobre probabilidad deficiente, puesto que el $50 \%$ no logra superar el $34 \%$ de las puntuaciones normadas. De acuerdo con los resultados de investigaciones previas, por ejemplo en Gómez (2014), estos profesores muestran tener un mayor conocimiento común del contenido que conocimiento avanzado del contenido y especializado.

Dado que el conocimiento didáctico-matemático sobre probabilidad no es observable de manera directa, hemos decidido inferirlo a partir de las respuestas de los participantes a las preguntas del Cuestionario CDM-Probabilidad, al ser observables siempre que la recopilación de datos sea completa y fiable (Godino, 1996). Es bajo esta mirada que se analizan algunas de las respuestas para cada una de las tres categorías globales de conocimiento sobre el contenido matemático que conforman el modelo CDM (Godino, 2009, Godino \& Pino-Fan, 2013). 


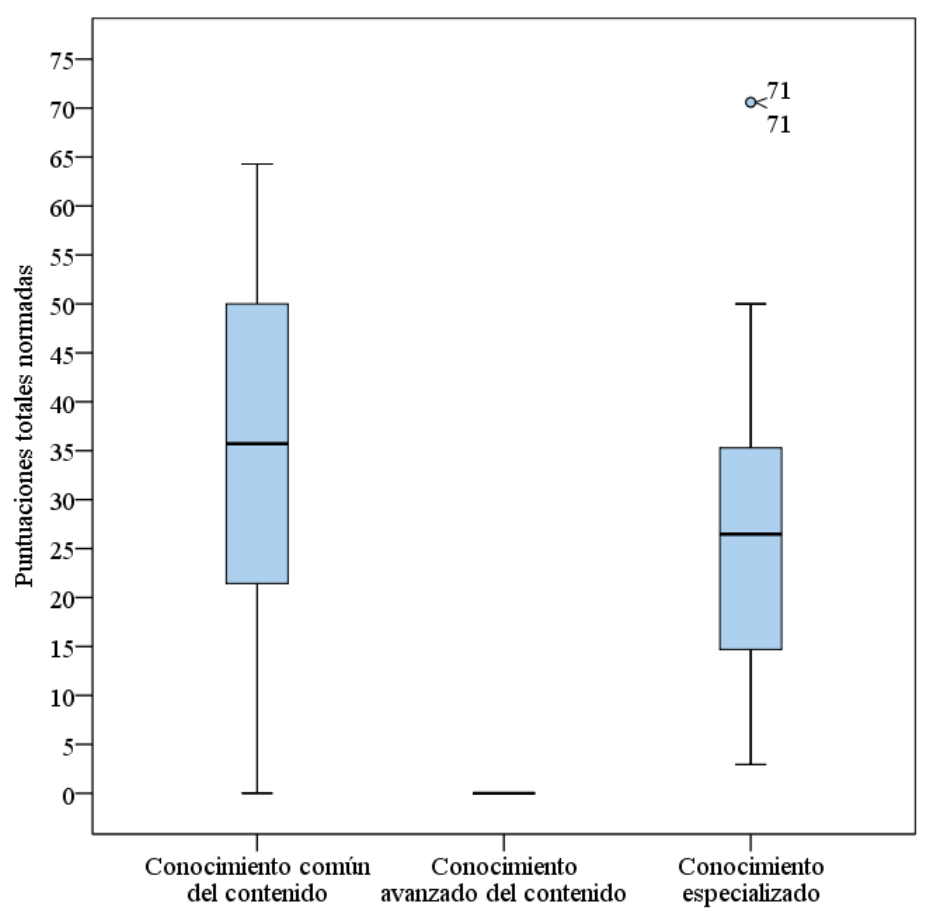

Figura 3. Distribución de puntajes normados según categorías del conocimiento didáctico-matemático.

Hemos complementado el análisis cuantitativo con otro cualitativo para obtener datos más detallados acerca de las dificultades de los participantes en el estudio. Para ello se procedió a leer las respuestas, para luego agruparlas y categorizarlas por medio de un proceso cíclico e inductivo, característico del análisis cualitativo de datos (Buendía, Colás \& Hernández, 1998).

Una vez establecidas las principales categorías de respuestas, se consideró un análisis de los conocimientos puestos en juego en las respuestas, así como los conflictos manifestados en las respuestas a los ítems que dan lugar a respuestas parcialmente correctas o incorrectas. A continuación se presenta el análisis de aquellos ítems que presentaron mayor dificultad para los profesores.

\subsubsection{Conocimiento común del contenido}

Los profesores presentan un conocimiento deficiente, y en algunos casos muy deficiente, en casi la totalidad de los ítems que evalúan este tipo de conocimiento. Dentro de ellos, el que presenta un menor porcentaje de respuestas correctas es el ítem 7 pregunta a). Este ítem, elaborado a partir de situaciones-problema expuestas en los libros de texto de Educación Primaria chilenos, evalúa el conocimiento del profesor sobre el cálculo de probabilidades y cómo este se encuentra, en algunos casos, vinculado a la independencia de sucesos.

En la Tabla 3 se aprecia que solo el 3,2\% de los profesores da una respuesta y argumento correcto a la pregunta a). Un porcentaje considerable $(63,4 \%)$ resuelve de manera incorrecta, mientras que un $14 \%$ resuelve correctamente pero en base a un argumento incorrecto, o bien no argumenta su respuesta. Los argumentos se presentan en la Tabla 4, en la que puede observarse que únicamente un 3,2\% de los profesores argumentó correctamente su respuesta, enfocándose en la independencia y en la equiprobabilidad de los sucesos. Un ejemplo de ello es la respuesta: "Si el dado tiene 
6 caras la probabilidad es la misma para cualquier número, y no varía la probabilidad la cantidad de números que ya han salido" (profesor 20). Como puede observarse, el argumento del profesor no se ve influenciado por la secuencia de resultados ya obtenidos.

Tabla 3. Frecuencias y porcentajes para el grado de corrección de las respuestas a la pregunta a).

\begin{tabular}{lcc}
\hline \multicolumn{1}{c}{ Grado de Corrección } & Frecuencia & Porcentaje \\
\hline Correcta & 3 & 3,2 \\
Parcialmente correcta & 13 & 14,0 \\
Incorrecta & 59 & 63,4 \\
No responde & 18 & 19,4 \\
Total & 93 & 100 \\
\hline
\end{tabular}

Un 14\% de los participantes logran identificar que todos los números del espacio muestral tienen igual probabilidad de salir, pero no argumentan su respuesta o bien el argumento empleado no es correcto. Un ejemplo de este tipo de respuesta es "Creo que todos los números tienen la misma probabilidad de salir" (profesor 74).

Respecto a las respuestas incorrectas, la Tabla 4 muestra que el argumento utilizado mayoritariamente $(29 \%)$ es considerar que en un nuevo lanzamiento del dado es más probable que salga el 2 o el 3, ya que estos números han salido mayor número de veces. Un ejemplo de este tipo de respuesta es la otorgada por el profesor 67: "El número que es más probable que salga puede ser el 2 y 3, ya que han salido más veces". En este tipo de respuesta se observa una fuerte influencia de la secuencia de resultados obtenidos en los lanzamientos anteriores, conflicto conocido recencia positiva, que ha sido descrito por Piaget e Inhelder (1951). En contraposición a este tipo de sesgo están los profesores $(20,4 \%)$ que presentan el sesgo de recencia negativa, es decir, que consideran que puesto que ya ha salido muchas veces el 2 y el 3 , ya es momento de que salga el 4 o el 6 , puesto que estos números han salido menos veces. Un ejemplo de este tipo de respuesta es: "El 4 o el 6, porque ha salido menos veces" (profesor 11).

Tabla 4. Frecuencias y porcentajes para los distintos tipos de respuestas a la pregunta a).

\begin{tabular}{lcc}
\hline \multicolumn{1}{c}{ Respuestas } & Frecuencia & Porcentaje \\
\hline $\begin{array}{l}\text { Todos los números del 1 al 6 tienen igual probabilidad de salir, } \\
\text { pues son sucesos equiprobables. }\end{array}$ & 3 & 3,2 \\
$\begin{array}{l}\text { Todos los números del 1 al 6 tienen igual probabilidad de salir. } \\
\text { Es más probable que salga el 2 o el 3 ya que han salido mayor } \\
\text { número de veces. }\end{array}$ & 13 & 14 \\
$\begin{array}{l}\text { Es más probable que salga el 4 o el 6 ya que han salido menos } \\
\text { veces. }\end{array}$ & 19 & 29 \\
$\begin{array}{l}\text { Es más probable que salga el 1 o el 5 ya que no ha salido en } \\
\text { ninguno de los lanzamientos anteriores. }\end{array}$ & 10 & 20,4 \\
$\begin{array}{l}\text { Otras respuestas y argumentaciones } \\
\text { No responde }\end{array}$ & 3 & 10,8 \\
Total & 93 & 3,2 \\
\end{tabular}

Por último, en los argumentos asociados a las respuestas incorrectas encontramos con una menor frecuencia $(10,8 \%)$ los que consideran como más probable que al lanzar el dado nuevamente, se obtenga un 1 o un 5, puesto que estos números no han salido en los lanzamientos anteriores, manifestando así el sesgo de recencia negativa. Un ejemplo de este tipo de respuesta es: "Como cada número tiene la misma 
probabilidad 1/6, y el número 1 y el número 5 no han salido aún, es más probable que salgan" (profesor 22).

Si observamos con detención esta respuesta, en el argumento aparece algo bastante curioso y es que pese a que el profesor reconoce que todos los números tienen la misma probabilidad de salir (lo que él indica como 1 es a 6) señala que es más probable que en un próximo lanzamiento salgan aquellos números que hasta el momento no han salido, es decir, el 1 y el 5. Aquí se aprecia la fuerte influencia que tiene el sesgo de recencia negativa en la comprensión de independencia de sucesos.

En relación a las respuestas clasificadas como "otras respuestas y argumentaciones", en ella incluimos respuestas que no tienen relación con la pregunta y que presentan argumentos sin sentido, tales respuestas corresponden a un $3,2 \%$.

\subsubsection{Conocimiento avanzado del contenido}

Para analizar el conocimiento avanzado del contenido sobre probabilidad, analizamos las respuestas obtenidas a la pregunta d) del ítem 7 del cuestionario CDMProbabilidad. La Tabla 5 evidencia que este ítem resultó de gran dificultad, pues ninguno de los profesores logró responder ni correctamente ni de forma parcialmente correcta. Tan solo el 34,4\% de los profesores respondió a la pregunta, sin embargo, lo hace de manera incorrecta.

Tabla 5. Frecuencias y porcentajes para el grado de corrección de las respuestas a la pregunta d).

\begin{tabular}{lcc}
\hline \multicolumn{1}{c}{ Grado de Corrección } & Frecuencia & Porcentaje \\
\hline Correcta & 0 & 0 \\
Parcialmente correcta & 0 & 0 \\
Incorrecta & 32 & 34,4 \\
No responde & 61 & 65,6 \\
Total & 93 & 100 \\
\hline
\end{tabular}

Al analizar los argumentos presentes en las respuestas incorrectas, los clasificamos como se muestra en la Tabla 6. Dicha tabla muestra que la mayoría de los profesores $(32,2 \%)$ se basa en argumentos erróneos, señalando que el tipo de problema planteado se puede relacionar con la probabilidad en problemas de mayor complejidad. Un ejemplo de este tipo de respuesta es el siguiente: "Considero que el contenido del lanzamiento de un dado se puede relacionar con la resolución de problemas de mayor complejidad presentes en el currículo, que requieran incorporar mayor número de variables" (profesor 93).

Tabla 6. Frecuencias y porcentajes para los distintos tipos de respuestas a la pregunta d).

\begin{tabular}{lcc}
\hline \multicolumn{1}{c}{ Respuestas } & Frecuencia & Porcentaje \\
\hline Probabilidad en problemas de mayor complejidad & 30 & 32,2 \\
Otras respuestas y argumentaciones & 2 & 2,2 \\
No responde & 61 & 65,6 \\
Total & 93 & 100 \\
\hline
\end{tabular}

En este tipo de respuesta, el profesor no logra identificar el contenido vinculado a la situación problemática planteada, ni tampoco con qué tipo de conceptos más avanzados del currículo se relaciona esta situación, pues solo considera al lanzamiento de dados como un elemento que puede ser utilizado para resolver "problemas de 
mayor complejidad". Por otro lado, un $2,2 \%$ de los profesores da respuestas sin sentido que clasificamos en la categoría "otras respuestas y argumentaciones".

\subsubsection{Conocimiento especializado}

Este estudio pretende indagar en algunos aspectos parciales o iniciales del conocimiento especializado, puesto que su exploración en profundidad requiere analizar aspectos como la idoneidad didáctica de las prácticas de los profesores en acción o de las argumentaciones empleadas en el proceso de enseñanza, aspectos a los que no es posible acceder por medio de las argumentaciones dadas a las preguntas que componen el cuestionario. Así, el análisis se centra en la reflexión epistémica que el profesorado es capaz de realizar sobre los conceptos y/o propiedades que se ponen en juego en la solución de la situación problemática planteada.

Los datos obtenidos evidencian una gran deficiencia en el conocimiento especializado sobre probabilidad, pues los porcentajes de respuestas correctas son, en general, muy bajos. Dentro de los ítems que evalúan este tipo de conocimiento, el que presentó un menor porcentaje de respuestas correctas es la pregunta c) del ítem 1, cuyo propósito es evaluar el conocimiento especializado en relación a la comprensión de independencia de sucesos en ensayos repetidos bajo las mismas condiciones de un experimento aleatorio.

En la Tabla 7 se observa que los profesores tuvieron grandes dificultades para resolver la situación problemática, pues ninguno logra identificar 4 o más conceptos y/o propiedades involucradas. Solo un 2,2\% logra identificar 2 o 3 conceptos y/o propiedades, mientras que un $72 \%$ solo un concepto y/o propiedad involucrada en la resolución del ítem 1.

Tabla 7. Frecuencias y porcentajes para el grado de corrección de las respuestas a la pregunta c).

\begin{tabular}{lcc}
\hline \multicolumn{1}{c}{ Grado de Corrección } & Frecuencia & Porcentaje \\
\hline Correcta & 0 & 0,0 \\
Parcialmente correcta & 2 & 2,2 \\
Incorrecta & 67 & 72,0 \\
No responde & 24 & 25,8 \\
Total & 93 & 100 \\
\hline
\end{tabular}

La Tabla 8 muestra los tipos de conceptos y/o propiedades que se encuentran presentes y ausentes en las respuestas de los profesores.

Como puede apreciarse, el concepto involucrado mayormente identificado es "probabilidad", con un 61,2\%, seguido por "regla de Laplace", con un 12,9\% y "espacio muestral", con un 7,5\%. Un ejemplo sería: "Cálculo de probabilidades, espacio muestral, regla de Laplace..." (profesor 60).

Si bien la noción de simetría de la moneda no fue mencionada explícitamente, se deduce a partir de las respuestas de un 4,3\% de los profesores. Un ejemplo de este tipo de respuesta es: "Las dos posibilidades, cara o sello, porque es una moneda honesta y hay igual probabilidad" (profesor 87). En esta respuesta vemos que el profesor da a entender que dado que el espacio muestral se encuentra conformado por dos posibles resultados, ambos sucesos tienen igual probabilidad de ocurrir. 
Tabla 8. Conceptos y/o propiedades identificadas por los profesores en la resolución de la situación problemática planteada en el ítem 1.

\begin{tabular}{|c|c|c|}
\hline Tipos de conceptos y/o propiedades identificados & Frecuencia & Porcentaje \\
\hline Experimento aleatorio & 0 & 0 \\
\hline Espacio muestral & 7 & 7,5 \\
\hline Probabilidad & 29 & 31,1 \\
\hline Regla de Laplace & 12 & 12,9 \\
\hline Equiprobabilidad & 3 & 3,2 \\
\hline Simetría de la moneda & 4 & 4,3 \\
\hline Independencia de sucesos & 0 & 0 \\
\hline $\begin{array}{l}\text { Otros (fracciones, porcentajes, proporciones, } \\
\text { frecuencia, etc.) }\end{array}$ & 18 & 19,4 \\
\hline No responde & 24 & 25,8 \\
\hline
\end{tabular}

La equiprobabilidad de sucesos es mencionada por un 3,2\% de los profesores. Un ejemplo sería: "Determinar equiprobabilidad de sucesos teóricamente en diversos experimentos" (profesor 46).

Dentro de las respuestas que incluimos en la categoría "otros" están aquellas que identifican conceptos que no guardan relación directa con los que se buscaba evidenciar mediante la situación planteada. Un ejemplo de esto es la siguiente respuesta: "Fracciones, multiplicación, porcentaje" (profesor 64).

Por último, hay que señalar que el experimento aleatorio e independencia de sucesos, conceptos centrales detrás de la situación problemática, no fueron identificados por ninguno de los profesores.

\section{Reflexiones finales}

En este estudio evaluamos de manera fiable el conocimiento didácticomatemático para la enseñanza de la probabilidad que poseen los profesores de Educación Primaria en activo. Los resultados evidencian un conocimiento muy insuficiente, pues la media de las puntuaciones totales es de 10,92 puntos de un total de 44 puntos posibles. Además, se observan serias dificultades para resolver correctamente las situaciones problemáticas, manifestándose variados errores y dificultades, como la presencia de heurísticas y sesgos probabilísticos asociados a la recencia negativa y positiva (Tversky \& Kahneman, 1982), al igual que en los resultados obtenidos por Batanero et al. (2005), Gómez (2014) y por Batanero et al. (2015) con futuros profesores de primaria.

Si comparamos los resultados obtenidos en nuestro estudio con los de Cañizares (1997) a partir de una muestra de niños de 10 a 14 años a los que se les plantearon problemas similares, se observa que los resultados de los participantes de nuestra investigación son muy inferiores. Esta situación es alarmante, si consideramos que en nuestro caso se trata de profesores de primaria en activo, que podrían transmitir tales sesgos a sus estudiantes.

Si nos concentramos en los componentes del conocimiento didáctico-matemático, observamos que el conocimiento común del contenido sobre probabilidad resulta ser de un nivel ligeramente mejor que el conocimiento avanzado del contenido y el conocimiento especializado. No obstante, el nivel de desempeño obtenido por este grupo de profesores chilenos ha sido demasiado bajo para todos los tipos de conocimientos involucrados. 
En síntesis, pues, el estudio otorga información que es de gran interés sobre todo para el contexto chileno, al proporcionar resultados originales sobre el conocimiento didáctico-matemático en relación a la probabilidad de los profesores de Educación Primaria en activo, puesto que en Chile prácticamente no existen investigaciones que den a conocer las necesidades de formación de este sector. Estos resultados globales sustentan la necesidad de realizar un programa de intervención que permita mejorar el nivel de los conocimientos matemáticos y didácticos para enseñar probabilidad del profesorado de Educación Primaria en activo.

\section{Referencias bibliográficas}

Azcárate, P., Cardeñoso, J. M., \& Porlán, R. (1998). Concepciones de futuros profesores de primaria sobre la noción de aleatoriedad. Enseñanza de las Ciencias, 16(1), 85-97.

Batanero, C., Godino, J. D., \& Cañizares, M. J. (2005) Simulation as a tool to train Preservice School Teachers. En J. Addler (Ed.), Proceedings of ICMI First African Regional Conference. Johannesburgo: International Commission on Mathematical Instruction.

Batanero, C., Gómez, E., Contreras, J. M. \& Díaz, C. (2015). Conocimiento matemático de profesores de primaria en formación para la enseñanza de la probabilidad: Un estudio exploratorio. Práxis Educativa 10(1). Recuperado de: http:// http://www.revistas2.uepg.br/index.php/praxiseducativa/article/view/6007.

Batanero, C., Gómez, E., Serrano, L., \& Contreras, J. M. (2012). Comprensión de la aleatoriedad por futuros profesores de educación primaria. Redimat, 1(3), 222-245.

Begg, A. \& Edwards, R. (1999). Teachers' ideas about teaching statistics. Proceedings of the 1999 combined conference of the Australian Association for Research in Education and the New Zealand Association for Research in Education. Melbourne: AARE \& NZARE. Recuperado de: http://www.aare.edu.au/99pap/beg99082.htm.

Buendía, L., Colás, P. \& Hernández, F. (1997). Métodos de investigación en Psicopedagogía. Madrid: McGraw-Hill.

Cañizares, M. J. (1997). Influencia del razonamiento proporcional y de las creencias subjetivas en las intuiciones probabilísticas primarias. Tesis Doctoral. Universidad de Granada.

Common Core State Standards Initiative (2010). Common Core State Standards for Mathematics. Recuperado de: http://www.corestandards.org/assets/CCSSI_Math\%20Standards.pdf

Fischbein, E., \& Gazit, A. (1984). Does the teaching of probability improve probabilistic intuitions? Educational Studies in Mathematics, 15, 1-24.

Godino, J. D. (1996). Mathematical concepts, their meaning, and understanding. En, L. Puig y A. Gutierrez (Eds.), Proceedings of the 20th Conference of the International Group for the Psychology of Mathematics Education (pp. 2-417-424), Universidad de Valencia.

Godino, J. D. (2002). Un enfoque ontológico y semiótico de la cognición matemática. Recherches en Didactique des Mathématiques, 22(2/3), 237-284.

Godino, J. D. (2009). Categorías de análisis de los conocimientos del profesor de matemáticas. UNION, Revista Iberoamericana de Educación Matemática, 20, 13-31.

Godino, J. D., Batanero, C., \& Font, V. (2007). The onto-semiotic approach to research in mathematics education. ZDM, The International Journal on Mathematics Education, $39(1-2), 127-135$. 
Godino, J. D., \& Pino-Fan, L. (2013). The mathematical knowledge for teaching. A view from onto-semiotic approach to mathematical knowledge and instruction. En B. Ubuz, Ç. Haser \& M. Mariotti (Eds.), Proceedings of the Eighth Congress of European Research in Mathematics Education (pp. 3325-3326). Antalya, Turkey: CERME.

Godino, J. D., Gonzato, M., \& Fernández, L. (2010). ¿Cuánto suman los ángulos interiores de un triángulo? Conocimientos puestos en juego en la realización de una tarea matemática. En M.M. Moreno, A. Estrada, J. Carrillo, y T. A. Sierra (Eds.), Investigación en Educación Matemática XIV (pp. 341-352). Lleida: SEIEM.

Gómez, E. (2014). Evaluación y desarrollo del conocimiento matemático para enseñar la probabilidad en futuros profesores de educación primaria. Tesis Doctoral. Universidad de Granada.

Gómez, E., Batanero, C. \& Contreras, C. (2013). Conocimiento matemático de futuros profesores para la enseñanza de la probabilidad desde el enfoque frecuencial. Bolema, 28 (48), 209-229.

Green, D. (1983). A survey of probability concepts in 3000 pupils aged 11-16 years. Proceedings of the First International Conference on Teaching Statistics, 2, Teaching Statistics Trust. (pp. 766-783). Sheffield, UK: Teaching Statistics Trust.

Hill, H. C., Ball, D.L., \& Schilling, S.G. (2008). Unpacking pedagogical content knowledge: Conceptualizing and measuring teachers' topic-specific knowledge of students. Journal for Research in Mathematics Education, 39, 372-400.

Konold. C. (1991). Understanding students' beliefs about probability. En E. von Glasersfeld (Ed.), Radical constructivism in Mathematics Education (pp. 139-156). Dordrecht: Kluwer.

Lecoutre, M. P. (1992). Cognitive Models and Problem spaces in "Purely Random" Situations. Educational Studies in Mathematics, 23, 557-568.

Lecoutre, M. P. \& Durand, J. L. (1988). Jugéments probabilistes et modéles cognitifs: étude d'une situation aléatoire. Educational Studies in Mathematics, 19, 357-368.

Mohamed, N. (2012). Evaluación del conocimiento de los futuros profesores de educación primaria sobre probabilidad. Tesis Doctoral. Universidad de Granada.

Ministerio de Educación (2012). Bases Curriculares 2012: Educación Básica Matemática. Santiago de Chile: Unidad de Currículum y Evaluación.

Ministerio de Educación y Ciencia (2007). Boletín oficial del Estado. ORDEN ECI/2211/2007, del 20 de julio, por la que se establece el currículo y regula la ordenación de la Educación Primaria. Madrid: Ministerio de Educación y Ciencia.

Muñiz, J. (1994). Teoría clásica de los tests. Madrid: Pirámide.

National Council of Teachers of Mathematics (1989). Curriculum and Evaluation Standards for School Mathematics. Reston, VA: NCTM.

National Council of Teachers of Mathematics (2003). Principios y estándares para la educación matemática. Sevilla: Sociedad Andaluza de Educación Matemática Thales.

OCDE (2010). Síntesis Estudio Económico de Chile, 2010. Recuperado de: http://www.oecd.org/dataoecd/7/38/44493040.pdf

Ortiz, J. J., Mohamed, N., Batanero, C., Serrano, L., \& Rodríguez, J. (2006). Comparación de probabilidades en maestros en formación. En P, Bolea, M. J. Gonzáles \& M. Moreno (Eds.), Actas del X Simposio de la Sociedad Española de Investigación en Educación Matemática (pp. 268-276). Huesca: SEIEM.

Pereira-Mendoza, L. (2002). Would you allow your accountant to perform surgery? Implications for the education of primary teachers. En B. Phillips (Ed.), Proceedings of 
the Sixth International Conference on the Teaching of Statistics. Hawthorn, VIC: International Statistical Institute.

Piaget, J., e Inhelder, B. (1951). La genése de l'idée de hasard chez l'enfant. París: Presses Universitaires de France.

Pino-Fan, L., Godino, J.D., \& Font, V. (2013). Diseño y aplicación de un instrumento para explorar la faceta epistémica del conocimiento didáctico-matemático de futuros profesores sobre la derivada (Parte 1). REVEMAT, 8(2), 1-49.

Tversky, A., \& Kahneman, D. (1982). On the psychology of prediction. En D. Kahneman, P. Slovic \& A. Tversky (Eds.), Judgement under uncertainty: Heuristics and biases (pp. 6983). Cambridge, MA: Cambridge University Press.

Vásquez, C. y Alsina, Á. (en prensa). Conocimiento Didáctico-Matemático del Profesorado de Educación Primaria sobre Probabilidad: Diseño, Construcción y Validación de un Instrumento de Evaluación. BOLEMA.

Vásquez, C. y Alsina, Á. (2014). Enseñanza de la Probabilidad en Educación Primaria. Un Desafío para la Formación Inicial y Continua del Profesorado. Números, 85, 5-23.

Watson, J. M. (2001). Profiling teachers competence and confidence to teach particular mathematics topics: The case of chance and data. Journal of Mathematics Teacher Education 4(4), 305-337.

\section{Referencias de los autores}

Claudia Vásquez Ortiz, Pontificia Universidad Católica de Chile, sede Villarrica (Chile), cavasque@uc.cl.

Ángel Alsina Pastells, Universidad de Girona (España), angel.alsina@udg.edu. 


\section{Teachers' knowledge for teaching probability: A global analysis based on Didactic-Mathematical Knowledge Model}

Claudia Vásquez Ortiz, Pontificia Universidad Católica de Chile, sede Villarrica (Chile).

Ángel Alsina Pastells, Universidad de Girona (España).

The purpose of this research is to assess the depth of teacher's didacticmathematical knowledge for teaching Probability in elementary schools. It has lately gained relevance considering how this subject has been incorporated throughout the Chilean school curriculum (NCTM, 1989; NCTM, 2000; CCSSI, 2010; MEC, 2007; MINEDUC, 2012), which represents an undeniable challenge for educators who are currently teaching in the classrooms, especially for whom are working in elementary schools; the vast majority did not get any significant training regarding how to instruct Probability during their initial teaching education.

Research concerning to this subject are still lacking, especially in countries like Chile where there are not available measure tools for performing a proper assessment of the different components of the didactic-mathematical knowledge. Therefore, in order to reach our goal we decided to create an instrument of assessment which allows us to delve into this topic in greater depth to obtain useful information for designing action plans.

The research has been conducted by adopting the Didactic-Mathematical Knowledge Model (Godino, 2009; Godino \& Pino-Fan, 2013; Pino-Fan, Godino \& Font, 2013), which is based on the Onto-Semiotic Approach (Godino, 2002; Godino, Batanero \& Font, 2007) to knowledge and mathematics education and provides a coherent set of tools to investigate about the didactic-mathematical knowledge of current elementary school's teachers for teaching Probability.

Under this perspective, a measure instrument called 'Questionnaire CDMProbability' was designed. The questionnaire was distributed to a sample of 93 Chilean elementary school's teachers. The feedback received were quite clear regarding the teacher's lack of didactic-mathematical knowledge in all of their components (common content knowledge, specialized content knowledge and extended content knowledge and their subcategories), because they didn't reach more than a $23 \%$ of correct answers in any of the mentioned categories. Furthermore, several heuristic and biased answers have demonstrated an insufficient understanding of Probability concepts. Then, the results of the measurement performed have proven that Chilean elementary school's teachers didn't acquire a proper knowledge in order to teach elementary students on a successful way

In accordance with the data obtained from the Questionnaire CDM-Probability', the main conclusions are to overcome the improvement of the didactic-mathematical knowledge of current elementary school's teachers for teaching this topic, and to achieve a significant enhancement to the contents in textbooks used in Chilean elementary schools. 


\section{ANEXO 1. Cuestionario CDM-Probabilidad}

Ítem 1: La profesora Gómez plantea la siguiente situación a sus alumnos de sexto año básico:

Una persona lanza 8 veces la misma moneda, obteniendo en orden, los siguientes resultados: cara, sello, cara, sello, sello, sello, sello, sello. Si lanza la moneda por novena vez, ¿qué es más probable que pase en el noveno lanzamiento?

Algunos de los alumnos de la profesora Gómez dan las siguientes respuestas:

Luis: es más probable que salga cara, puesto que han salido demasiados sellos y ya es hora de que salga cara

Responda:
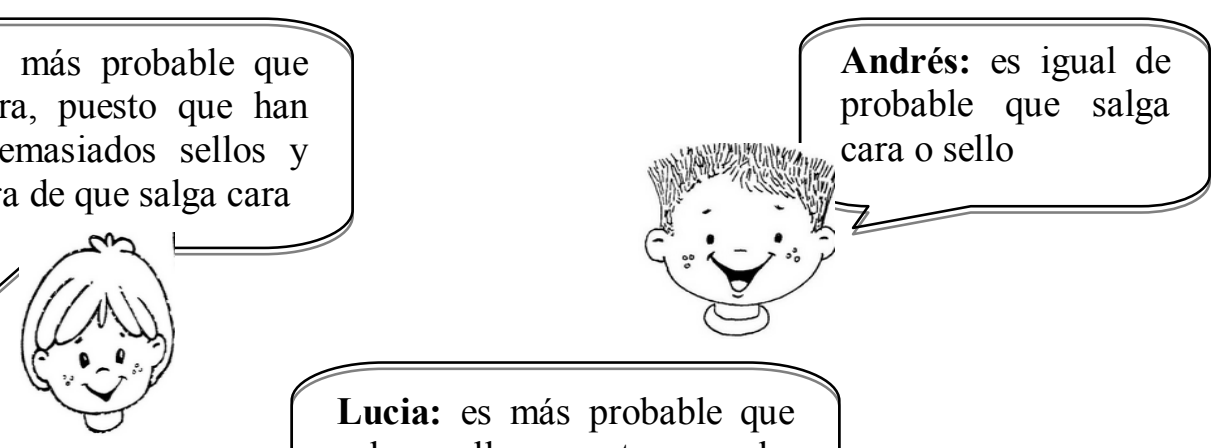

Lucia: es más probable que salga sello, puesto que ha salido sello en cinco

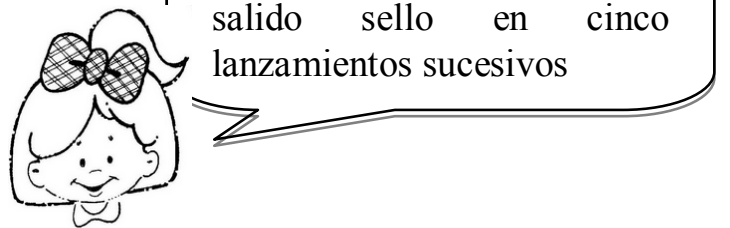

a. Resuelva el problema planteado por la profesora Gómez

b. ¿Cuál o cuáles de los alumnos ha dado con la respuesta correcta? ¿Por qué?

c. ¿Qué conceptos y/o propiedades matemáticas deben usar los alumnos para dar una solución correcta al problema planteado?

d. Describa las posibles dificultades, presentes en las respuestas incorrectas, que han llevado a los alumnos a responder de manera errónea.

e. ¿Qué estrategias utilizaría para ayudar a aquellos alumnos que no han sabido resolver el problema? Fundamente su respuesta.

Ítem 2: La profesora María Eugenia presenta el siguiente juego a sus alumnos:

Deben sacar una bola de una de las cajas siguientes con los ojos cerrados. Ganan si obtienen una bola blanca. ¿De qué caja es preferible hacer la extracción?

CAJA A

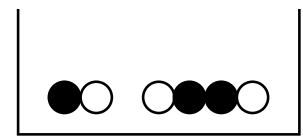

CAJA B

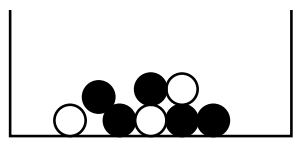




\section{Responda:}

a. Resuelva el problema

b. ¿Qué conceptos y/o propiedades matemáticas deben usar los alumnos para dar una solución correcta al problema planteado?

c. Describa las posibles dificultades, a las cuales podrían verse enfrentados los alumnos para resolver de manera correcta el problema.

d. ¿Qué estrategias utilizaría para ayudar a aquellos alumnos que no han sabido resolver el problema? Fundamente su respuesta.

Ítem 3: El profesor Ramírez plantea el siguiente problema a sus alumnos:

En una caja hay 4 bolas rojas, 3 verdes y 2 blancas. ¿Cuántas bolas se deben sacar para estar seguro de que se obtendrá una bola de cada color?

Las respuestas obtenidas por parte de algunos de sus alumnos son las siguientes:

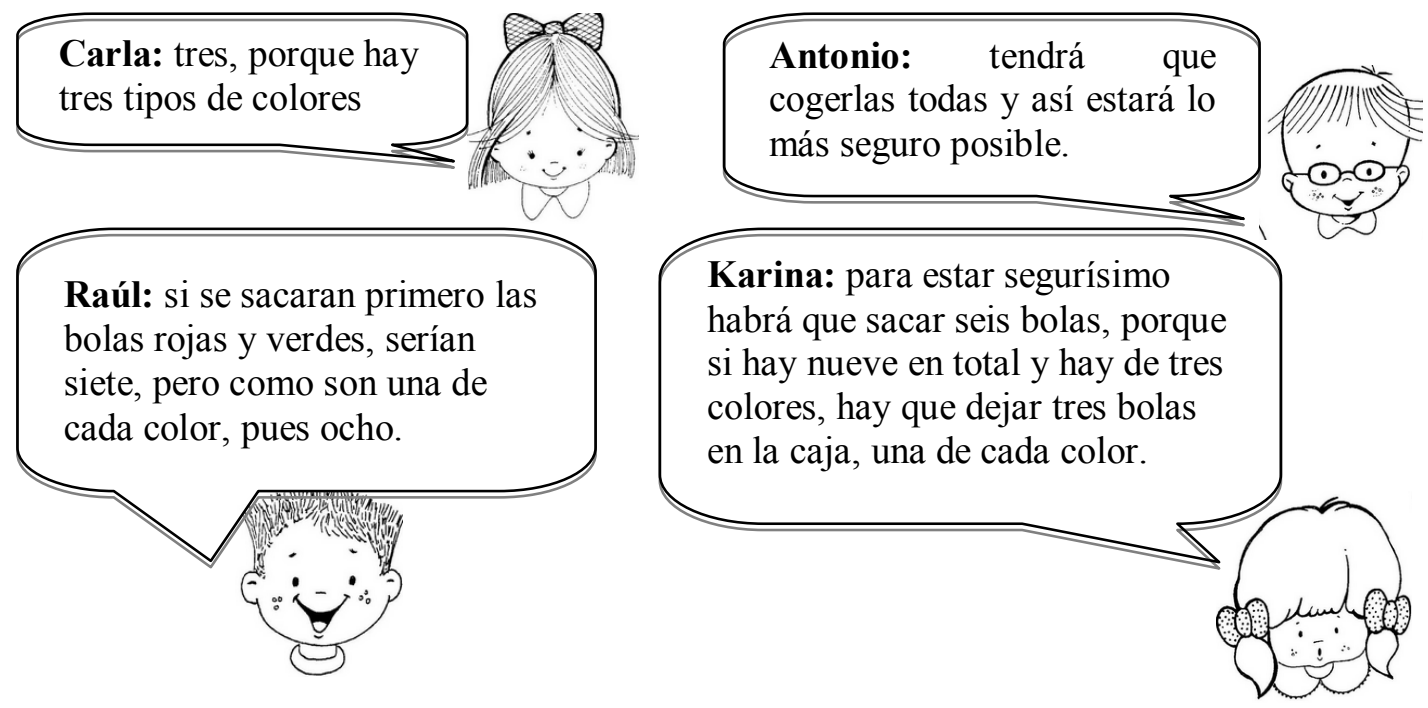

Responda:

a. ¿Qué respuestas debería aceptar el profesor como correctas? ¿Por qué?

b. ¿Qué conceptos y/o propiedades matemáticas deben usar los alumnos para dar una solución correcta al problema planteado?

c. ¿Qué estrategias utilizaría para que aquellos alumnos que han dado una respuesta errónea se den cuenta de su error y lo superen? Fundamente su respuesta.

Ítem 4: Usted se encuentra en quinto año básico y ha planteado el siguiente problema a sus alumnos:

En una clase de matemáticas hay 13 niños y 16 niñas. Cada alumno escribe su nombre en un trozo de papel y todos los trozos se ponen en un sombrero. El profesor saca uno sin mirar y pregunta a sus alumnos: ¿qué es más probable que suceda?

Uno de los alumnos da la siguiente respuesta: "Es la suerte quien decide. Aunque haya más niñas, la suerte es igual. En parte podría ganar una niña". 
Responda:

a. ¿Considera correcta la respuesta de este alumno? Justifique su veracidad o falsedad.

b. ¿Qué conceptos y/o propiedades deben usar los alumnos para dar una respuesta adecuada a este problema?

c. Describa las posibles dificultades, a las cuales podrían verse enfrentados los alumnos para resolver de manera correcta el problema.

d. ¿Qué estrategias utilizaría para que aquellos alumnos que han dado una respuesta errónea se den cuenta de su error y lo superen? Fundamente su respuesta.

Ítem 5: Pedro ha participado en una lotería semanal durante los dos últimos meses. Hasta ahora no ha ganado nunca, pero decide continuar por la siguiente razón:

"la lotería es un juego basado en la suerte, algunas veces gano, algunas veces pierdo. Yo ya he jugado muchas veces y nunca he ganado. Por lo tanto, estoy más seguro que antes de que ganaré en alguna partida próxima"

¿Cuál es su opinión sobre la explicación de Pedro?

Ítem 6: Eduardo tiene en su caja 10 bolas blancas y 20 negras. Luís tiene en su caja 30 bolas blancas y 60 negras. Juegan una partida de azar. El ganador es el niño que saque primero una bola blanca. Si ambos sacan simultáneamente una bola blanca o una bola negra, ninguno gana, devuelven las bolas a las cajas y la partida continua.

Eduardo afirma que el juego no es justo porque en la caja de Luís hay más bolas blancas que en la suya.

¿Considera correcta la respuesta de este alumno? Justifique su veracidad o falsedad.

Ítem 7: Usted ha seleccionado el siguiente problema para sus alumnos de $6^{\circ}$ básico:

Al lanzar un dado 10 veces han salido los siguientes valores: 3, 6, 2, 3, 4, 4, 3, 2, 6, 2. Si se lanza el dado otra vez, ¿qué número es más probable que salga?

Responda:

a. Resuelva el problema

b. ¿Qué objetivo cree usted que tiene, en relación al currículo, el abordar este tipo de problema?

c. ¿Qué tipo de recurso utilizaría para representar el problema? Explique cómo lo utilizaría. Justifique su elección.

d. ¿Con qué conceptos más avanzados del currículo escolar relaciona el contenido involucrado en la resolución de este problema? 